\title{
A case of linear morphoea mistaken for reflex sympathetic dystrophy
}

Steven Tien Guan Thng ${ }^{1}$, MBBS, MRCP, Keryi Wong ${ }^{2}$, BEng

ABSTRACT Morphoea, or localised scleroderma, is a disease entity with poorly understood pathogenesis. Early diagnosis of the condition is crucial in order to prevent permanent morbidity. However, initial presentations of morphoea can be nonspecific and easily mistaken for other conditions, resulting in late treatment and permanent disability. We report a case of linear morphoea in a 22-year-old man who was initially diagnosed with reflex sympathetic dystrophy. By the time the diagnosis of morphoea was confirmed, the patient had already developed contractures.

Keywords: contractures, linear morphoea, reflex sympathetic dystrophy

\section{INTRODUCTION}

Morphoea, also known as localised scleroderma, is a relatively rare disease characterised by excessive collagen deposition, leading to thickening of the dermis or subcutaneous tissues. It results from an imbalance between collagen deposition and destruction, but the disease's exact pathogenesis is not entirely understood. The prevalence of morphoea is equal in children and adults. Although the clinical course of this disease is benign, widespread fibrosis with resultant disabling contractures may lead to significant morbidity and complications. As such, it is crucial to promptly diagnose linear morphoea, as early intervention can prevent such complications. This report highlights a case of linear morphoea that was initially diagnosed as reflex sympathetic dystrophy. Linear morphoea was subsequently identified and treated, but treatment was complicated by disabling contractures.

\section{CASE REPORT}

A 22-year-old healthy, Chinese, male fighter pilot with no significant medical history presented with thickening of the skin, discolouration of the left arm and contractures of the fingers over a period of ten months. He initially presented to an orthopaedic surgeon with complaints of a tightening sensation over his triceps. As he gave a history of a possible mild injury (without fracture) during a golf game, he was diagnosed with reflex sympathetic dystrophy and treated with painkillers and physiotherapy. There was, however, no history of pain or burning sensation, or any history suggestive of vasomotor instability. Over the next ten months, the patient's condition worsened despite active physiotherapy. He developed significant fibrosis over a linear band of skin that extended from his triceps to his wrist, resulting in contractures of the fingers.

On physical examination at presentation, a linear strip of hypopigmentation on the dorsal side of the patient's left arm (Figs. 1 \& 2) was seen. Contractures of his left middle, ring and

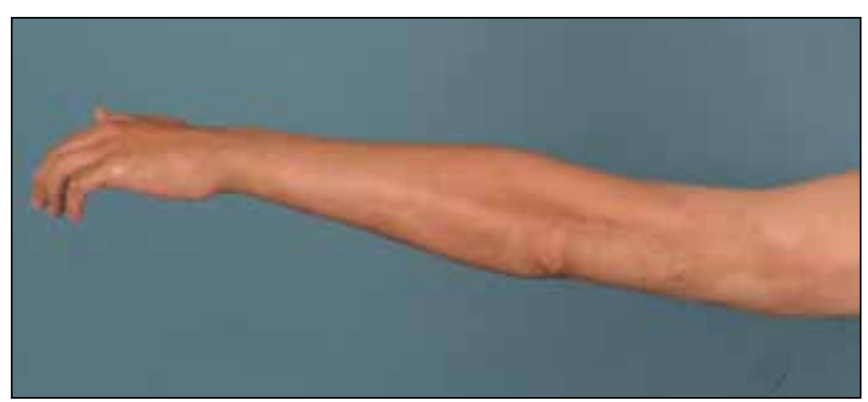

Fig. 1 Photograph shows a linear strip of hypopigmentation on the dorsal surface of the left arm.

little fingers were observed (Fig. 3). The skin over his left arm was indurated and fibrosed, but not tender. There was no abnormal skin moisture, or other extracutaneous or musculoskeletal signs. There were also no signs suggestive of systemic sclerosis. With a working diagnosis of linear morphoea, punch biopsies were taken over two sites at the border of the induration on the patient's left arm. Histopathological features from the biopsy confirmed the diagnosis of linear morphoea. Staining for complement and antibodies did not show any evidence of vasculitis, autoimmune diseases or lichenoid tissue reaction. Laboratory studies included tests for antibody markers, full blood count and erythrocyte sediment rate. All were within normal limits, except for a mildly raised erythrocyte sediment rate of $13 \mathrm{~mm} / \mathrm{hr}$.

With a confirmed diagnosis of linear morphoea, our patient was started on topical protopic and clobetasol, and concurrently treated with ultraviolet A1 (UVA1) phototherapy twice a week. After six months of treatment, his condition stabilised with no further progression of his fibrosis. There was also a slight improvement in the flexibility of his elbow and finger movements. As a fighter pilot then, the patient was unwilling to consider taking hydroxychloroquine, as it might affect his vision and consequently, his career.

${ }^{1}$ National Skin Centre, ${ }^{2}$ Duke-NUS Graduate Medical School, Singapore

Correspondence: Dr Steven Thng, Consultant, National Skin Centre, 1 Mandalay Road, Singapore 308205. steventhng@nsc.gov.sg 

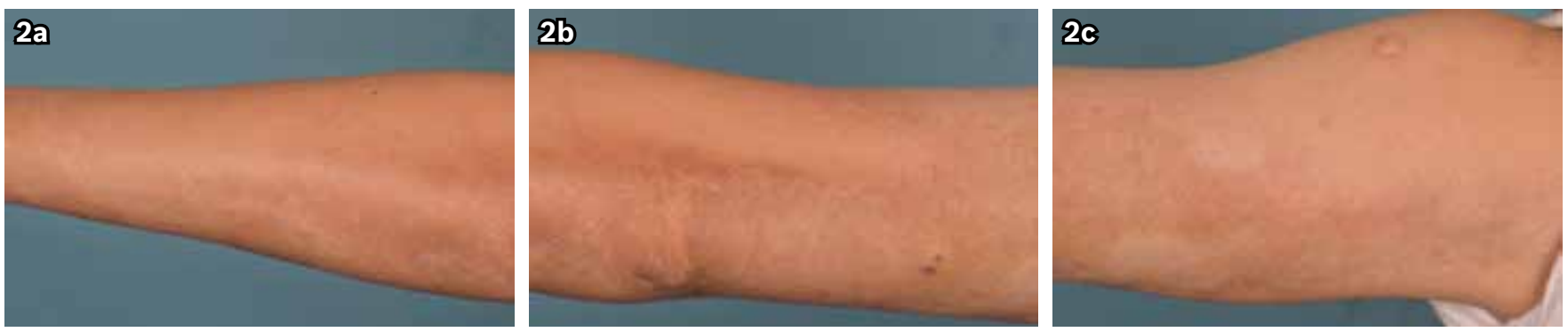

Fig. 2 Close-up photographs show hypopigmentation of the left (a) lower arm; (b) elbow; and (c) upper arm.

\section{DISCUSSION}

Also known as localised scleroderma, morphoea is a rare condition with an incidence of 0.4-2.7 per 100,000 people. ${ }^{(1,2)}$ It is most often found in children aged 2-14 years old ${ }^{(3)}$ and in adults aged 20-40 years old. ${ }^{(4)}$ The pathogenesis of morphoea is not entirely clear, but is presumably multifactorial, with contributions from autoimmunity, genetics and the environment. Reports of a significantly higher prevalence of concomitant autoimmune diseases such as vitiligo, insulin-dependent diabetes mellitus and psoriasis, as well as raised autoantibody titres among children and adults with morphoea, support an autoimmune pathogenesis of the disease. ${ }^{(3,5)}$ However, the clinical significance of raised autoantibodies has yet to be identified. In a retrospective review of 750 children with morphoea, Zulian et al found that the prevalence of autoimmune familial disease in these children was higher than in the general population, suggesting the presence of a genetic disposition. ${ }^{(6)}$ Environmental factors reported include trauma, infections and radiation. ${ }^{(5)}$ Autoimmune and environmental factors are thought to result in vascular injury, which causes the release of cytokines, leading to the activation of a cascade of cytokines. ${ }^{(5)}$ The upregulation of such cytokines causes a net deposition of collagen, which defines morphoea. ${ }^{(5)}$

There are several ways in which morphoea can manifest circumscribed, generalised, linear (includes en coup de sabre), pansclerotic or mixed. ${ }^{(7)}$ Regardless of subtype, all morphoea lesions undergo a transition from an initial inflammatory stage to sclerosis, and finally, to an atrophic stage. ${ }^{(5)}$ Most patients present with an erythematous patch or plaque, and some may complain of pain or itch at the site before the lesion is noticeable. The sclerotic stage may be accompanied by hypo- or hyperpigmentation, alopecia or a shiny cutaneous surface. Over time, the sclerotic lesions soften into hypo- or hyperpigmented plaques. Depending on the depth of involvement, the patient may be left with shallow or cigarette, paper-like depressions (indicating dermis involvement), or deep indentations (indicating involvement of subcutaneous tissue and beyond). In the case of linear morphoea, involvement of deep tissues can result in muscle weakness and contractures, as seen in our patient.

Reflex sympathetic dystrophy, however, is a clinical syndrome of unknown cause characterised by pain, swelling and vasomotor dysfunction. It is usually triggered by trauma, even though the trauma sustained may be trivial and insignificant. The distinct feature of reflex sympathetic dystrophy is pain (usually constant and disproportionate to the injury) that affects the whole limb

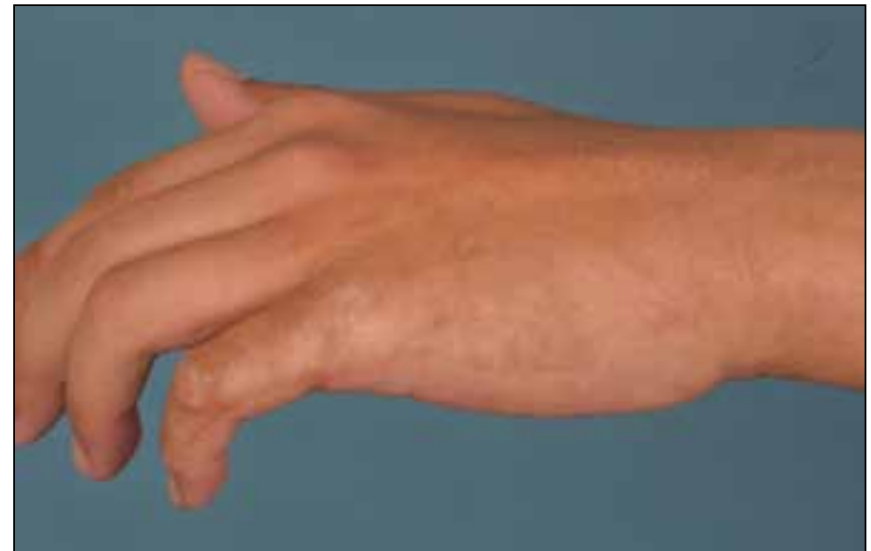

Fig. 3 Photograph shows contractures of the left middle, ring and last fingers.

rather than a small linear strip of skin. In addition, there are usually features of vasomotor instablility (e.g. increased sweating, cold hands) and oedema of the extremity - all of which were absent in our patient. Clinical features common to both late-stage reflex sympathetic dystrophy and linear morphoea include atrophy of the skin, fibrosis and contractures. However, involvement of only a linear strip of skin instead of the whole limb is a feature that is more consistent with linear morphoea. Other differential diagnoses of morphoea include scleroderma and eosinophilic fasciitis (EF). While sclerodactyly is a prominent feature of scleroderma, it is absent in morphoea. Although EF is also characterised by skin induration and erythema, the affected skin in EF exhibits a more woody, peau d'orange texture, as opposed to the typical shiny, smooth surface of skin affected by morphoea. EF also tends to spare the epidermis and superficial dermis, and is associated with eosinophilia.

Morphoea is a self-limiting disease. The active stage, which is characterised by inflammation, is most responsive to therapy. Sclerotic lesions are less likely to improve and atrophic lesions rarely respond to therapy. Thus, the expected outcome of therapy is most often a reduction in erythema, softening of lesions or arrest of progressive disease, rather than restoration to normal skin. Therapeutic options include topical or systemic pharmacologics and phototherapy. Topical therapies are typically limited for use in diseases that do not extend beyond the dermis. Topical tacrolimus is useful in the treatment of active inflammatory disease. Clinical improvements in dyspigmentation, erythema and induration have been associated with the use of topical vitamin $\mathrm{D}^{(8)}$ and imiquimod, ${ }^{(9)}$ which may work via an 
inhibitory effect on T-lymphocyte activation ${ }^{(8)}$ and an increase in interferon-gamma (inhibits transforming growth factor beta and production of the extracellular matrix), ${ }^{(9)}$ respectively. Phototherapy with UVA1 $(340-400 \mathrm{~nm})$ has been presumed to work via the upregulation of the production of matrix metalloproteinases by fibroblasts that degrade collagen, and also via the immunomodulation of cytokines. ${ }^{(10)}$ The efficacy of UVA1 phototherapy in the treatment of morphoea is widely agreed upon. ${ }^{(11)}$ Systemic therapy with methotrexate and corticosteroids is used in patients with severe disease. ${ }^{(11)}$ However, the rarity of morphoea and the scarcity of validated outcome measures limit the availability of evidence-based treatment options for this disease.

Besides managing the existing disease, monitoring for extracutaneous manifestations and early referral are also pertinent, for example, it is important to check for arthritis and neurologic involvement in patients with head and neck morphoea. Patients with disease in the extremities and face are at high risk of developing functional disabilities. Physical therapy may help to alleviate the progression of movement impairments. Although active morphoea usually lasts for 3-6 years, reactivation can occur after periods of remission.

This case demonstrates the importance of early recognition of features of linear morphoea so that treatment may be instituted early to prevent progression of the condition to disabling contractures, which unfortunately, happened in our case. While the presentation of linear morphoea and reflex sympathetic dystrophy may be similar in some aspects, the absence of pain, swelling and vasomotor instability, coupled with the involvement of only a small part of the extremity, should alert clinicians to the diagnosis of linear morphoea. A biopsy should be done for early confirmation of the diagnosis and institution of treatment.

\section{REFERENCES}

1. Peterson LS, Nelson AM, Su WP, et al. The epidemiology of morphea (localized scleroderma) in Olmsted County 1960-1993. J Rheumatol 1997; 24:73-80.

2. Murray KJ, Laxer RM. Scleroderma in children and adolescents. Rheum Dis Clin North Am 2002; 28:603-24.

3. Leitenberger JJ, Cayce RL, Haley RW, et al. Distinct autoimmune syndromes in morphea: a review of 245 adult and pediatric cases. Arch Dermatol 2009; 145:545-50.

4. Sehgal VN, Srivastava G, Aggarwal AK, et al. Localized scleroderma/ morphea. Int J Dermatol 2002; 41:467-75.

5. Fett N, Werth VP. Update on morphea: part I. Epidemiology, clinical presentation, and pathogenesis. J Am Acad Dermatol 2011; 64:217-28.

6. Zulian F, Athreya BH, Laxer R, et al. Juvenile localized scleroderma: clinical and epidemiological features in 750 children. An international study. Rheumatology (Oxford) 2006; 45:614-20.

7. Laxer RM, Zulian F. Localized scleroderma. Curr Opin Rheumatol 2006; 18:606-13.

8. Vilela FA, Carneiro S, Ramos-e-Silva M. Treatment of morphea or localized scleroderma: review of the literature. J Drugs Dermatol 2010; 9:1213-9.

9. Dytoc M, Ting PT, Man J, Sawyer D, Fiorillo L. First case series on the use of imiquimod for morphoea. Br J Dermatol 2005; 153:815-20.

10. Kroft EB, Berkhof NJ, van de Kerkhof PC, Gerritsen RM, de Jong EM. Ultraviolet A phototherapy for sclerotic skin diseases: a systematic review. J Am Acad Dermatol 2008; 59:1017-30.

11. Fett N, Werth VP. Update on morphea: part II. Outcome measures and treatment. J Am Acad Dermatol 2011; 64:231-42. 